Grant/research support from: AbbVie, MSD, Novartis, and Pfizer, Consultant of: AbbVie, Bristol-Myers Squibb, Eli Lilly, MSD, Novartis, Pfizer, Roche, UCB, Speakers bureau: AbbVie, Bristol-Myers Squibb, Eli Lilly, MSD, Novartis, Pfizer, Roche, UCB, Thibaud Coroller Shareholder of: Novartis, Employee of: Novartis, Brian Porter Shareholder of: Novartis, Employee of: Novartis, Shephard Mpofu Shareholder of: Novartis, Employee of: Novartis, Aimee Readie Shareholder of: Novartis, Employee of: Novartis DOI: 10.1136/annrheumdis-2020-eular.1207

\section{OP0061 FEASIBILITY STUDY ON AN AUTOMATED QUANTITATIVE SYSTEM FOR ULTRASOUND JOINT INFLAMMATION ASSESSMENT IN RHEUMATOID ARTHRITIS USING DEEP LEARNING}

Y. K. $\operatorname{Tan}^{1}$, S. Suriyanto ${ }^{2}$, P. H. Yeung ${ }^{3}$, S. Xu $\mathbf{u}^{4,5} .{ }^{1}$ Department of Rheumatology and Immunology, Singapore General Hospital, Singapore, Singapore; ${ }^{2}$ Diagnostics Development Hub, Accelerate Technologies, Singapore, Singapore; ${ }^{3}$ Department of Engineering Science, Institute of Biomedical Engineering, University of Oxford, Oxford, United Kingdom; ${ }^{4}$ Department of General Surgery, Nanfang Hospital, Southern Medical University, Guangzhou, China; ${ }^{5}$ Voxel Imaging Pte Ltd, Singapore, Singapore

Background: The most widely accepted ultrasound (US) joint inflammation scoring system in rheumatoid arthritis (RA) is semi-quantitative in nature. This process involves manual image acquisition followed by image interpretation. The subjectivity inherent in manual scoring may be overcome by the development of an automated quantitative system to measure joint inflammation.

Objectives: To develop an automated quantitative system to measure US detected power Doppler (PD) joint inflammation in patients with RA.

Methods: The synovial region of interest (sROI) on US images at the metacarpophalangeal joints (MCPJs) and the metatarsophalangeal joint (MTPJs) within the Doppler box is manually segmented by a clinician experienced in musculoskeletal US (figure 1). PD joint inflammation was scored manually semi-quantitatively (0-3). Deep learning based image segmentation was applied to the US images to automatically identify sROI and quantify the amount of PD signals within the SROI (figure 1) to obtain a computer derived PD reading reflecting the extent of PD vascularity within the sROI. The performance of computer derived PD reading was evaluated in comparison with the clinician's manual scoring.

Results: 820 joints from bilateral $1^{\text {st }}$ to $5^{\text {th }}$ MCPJs and MTPJs in 41 adult RA patients (baseline characteristics: $75.6 \%$ Chinese; $73.2 \%$ female; mean (SD) DAS28, 4.23 (1.25); mean (SD) disease duration, 73.3 (57.8) months) were evaluated in this cross-sectional study. The respective mean (SD)/ median (IQR) computer derived PD readings were $0.13(0.75) / 0.04(0.08), 1.62(1.77) / 1.21$ (1.19) and $10.12(6.86) / 7.51$ (5.24) for manual score 0,1 and 2 (no joints had manual score 3), with statistically significant differences found among the different manual score classes (for non-normally distributed data, Kruskal-Wallis

Figure 1. Manual and automated segmentation of synovial region of interest

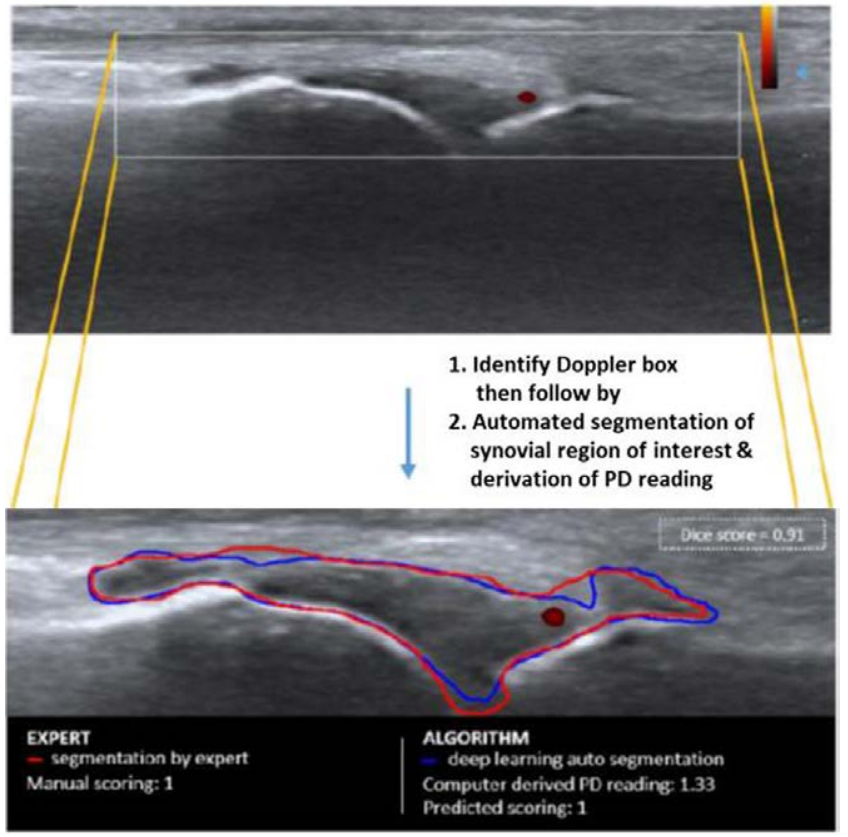

Table 1. Performance of computer prediction versus clinician evaluation

\section{Score 0 vs. 1}

Assessor

Computer Prediction: Score 0

Computer Prediction: Score 1

Sensitivity $=99.14 \%$,

Specificity $=97.00 \%$

Score 1 vs. 2

Assessor

Computer Prediction: Score 1

Computer Prediction: Score 2

Sensitivity $=97.14 \%$

Specificity $=93.97 \%$
Clinician Evaluation: Score 0 Clinician Evaluation: Score 1 615
19
115

Clinician Evaluation: Score 1 Clinician Evaluation: Score 2

$\begin{array}{cc}109 & 2 \\ 7 & 68\end{array}$
H-test, $p=1.69 \times 10^{-92}$, Mann-Whitney Test: manual score 0 versus $1, p=1.04$ x $10^{-62}$; manual score 0 versus $2, p=3.28 \times 10^{-43}$; manual score 1 versus 2 , $\mathrm{p}=1.53 \times 10^{-28}$ ). Area under the ROC curve (AUC) based on computer derived PD reading cut-off of 0.26 to identify manual score 0 versus 1 was 0.98 , while AUC based on computer derived PD reading cut-off of 3.37 to identify manual score 1 versus 2 was 0.98 . The overall agreement of the score classes $(0,1$ and 2) based on computer prediction using the above cut-offs versus manual scores of 0,1 and 2 is $791 / 820=96.46 \%$. Table 1 summarizes the performance of computer prediction using the above cut-offs when compared to clinician evaluation (i.e. score 0 versus 1, comparing computer prediction with clinician evaluation, sensitivity $=99.14 \%$ and specificity $=97.00 \%$; score 1 versus 2 , comparing computer prediction with clinician evaluation, sensitivity $=97.14 \%$ and specificity=93.97\%).

Conclusion: An automated quantitative system for US PD joint inflammation assessment using deep learning showed high sensitivity and specificity when results from computer prediction were compared to clinician evaluation. Further validation in a larger RA cohort with a longitudinal study design would be required.

References: Nil

Disclosure of Interests: None declared

DOI: 10.1136/annrheumdis-2020-eular.1501

\section{OP0062 PREDICTIVE VALUE OF BONE TEXTURE FEATURES EXTRACTED BY DEEP LEARNING MODELS FOR THE DETECTION OF OSTEOARTHRITIS: DATA FROM THE OSTEOARTHRITIS INITIATIVE}

C. F. Kuo ${ }^{1,2}$, K. Zheng ${ }^{3}$, S. Miao ${ }^{3}$, L. Lu ${ }^{3}$, C. I. Hsieh ${ }^{1}$, C. Lin ${ }^{2}$, T. Y. Fan ${ }^{2} .{ }^{1}$ Chang Gung Memorial Hospital, Division of Rheumatology, Allergy and Immunology, Taoyuan, Taiwan, Republic of China; ${ }^{2}$ Chang Gung Memorial Hospital, Center for Artificial Intelligence in Medicine, Taoyuan, Taiwan, Republic of China; ${ }^{3} \mathrm{PA} / \mathrm{I}$ labs, Bethesda, United States of America

Background: Osteoarthritis is a degenerative disorder characterized by radiographic features of asymmetric loss of joint space, subchondral sclerosis, and osteophyte formation. Conventional plain films are essential to detect structural changes in osteoarthritis. Recent evidence suggests that fractal- and entropy-based bone texture parameters may improve the prediction of radiographic osteoarthritis. ${ }^{1}$ In contrast to the fixed texture features, deep learning models allow the comprehensive texture feature extraction and recognition relevant to osteoarthritis.

Objectives: To assess the predictive value of deep learning-extracted bone texture features in the detection of radiographic osteoarthritis.

Methods: We used data from the Osteoarthritis Initiative, which is a longitu dinal study with 4,796 patients followed up and assessed for osteoarthritis. We used a training set of 25,978 images from 3,086 patients to develop the textual model. We use the BoneFinder software ${ }^{2}$ to do the segmentation of distal femur and proximal tibia. We used the Deep Texture Encoding Network (Deep-TEN) ${ }^{3}$ to encode the bone texture features into a vector, which is fed to a 5-way linear classifier for Kellgren and Lawrence grading for osteoarthritis classification. We also developed a Residual Network with 18 layers (ResNet18) for comparison since it deals with contours as well. Spearman's correlation coefficient was used to assess the correlation between predicted and reference $\mathrm{KL}$ grades. We also test the performance of the model to identify osteoarthritis ( $\mathrm{KL}$ grade $\geq 2$ ).

Results: We obtained 6,490 knee radiographs from 446 female and 326 male patients who were not in the training sets to validate the performance of the models. The distribution of the $\mathrm{KL}$ grades in the training and testing sets were shown in Table 1. The Spearman's correlation coefficient was 0.60 for the Deep-TEN and 0.67 for the ResNet18 model. Table 2 shows the performance of the models to detect osteoarthritis. The positive predictive value for Deep-TEN and ResNet18 model classification for OA was $81.37 \%$ and $87.46 \%$, respectively. 